\title{
Chronic modulation of AMP-Kinase, Akt and mTOR pathways by ionizing radiation in human lung cancer xenografts
}

Yaryna Storozhuk ${ }^{1,3}$, Toran Sanli ${ }^{1,4}$, Sarah N Hopmans ${ }^{4}$, Carrie Schultz ${ }^{2}$, Tom Farrell ${ }^{2,6}$, Jean-Claude Cutz ${ }^{4}$, Gregory R Steinberg ${ }^{5}$, James Wright ${ }^{2,3}$, Gurmit Singh ${ }^{2,4}$ and Theodoros Tsakiridis ${ }^{1,2,3^{*}}$

\begin{abstract}
Introduction: Earlier, we showed that in cancer cells, AMP-activated kinase (AMPK) participates in a signal transduction pathway involving ATM-AMPK-p53/p2 $1^{\text {cip } 1}$ which is activated by ionizing radiation (IR) to mediate G2-M arrest and enhanced cytotoxicity. We also observed that AMPK modulates ATM expression and activity and the IR response of the Akt-mTOR pathway. Since the ATM, AMPK and Akt pathways are key targets of novel radio-sensitizing therapeutics, we examined the chronic modultion of expression and activity of those pathways by IR alone in xenograft models of lung cancer.
\end{abstract}

Methods: Immuno-compromised mice were grafted with human lung A549 and H1299 cells, were treated with a single fraction of 0 or $10 \mathrm{~Gy}$, and left to grow for 8 weeks. Extracted tumors were subjected to lysis and immunoblotting or fixation and immunohistochemical analysis.

Results: IR inhibited significantly xenograft growth and was associated with increased expression of Ataxia Telengiectasia Mutated (ATM) and enhanced phosphorylation of two ATM targets, H2Ax and checkpoint kinase Chk2. Irradiated tumours showed increased total AMPK levels and phosphorylation of AMPK and its substrate Acetyl-CoA Carboxylase (ACC). IR led to enhanced expression and phosphorylation of p53 and cyclin dependent kinase inhibitors $\mathrm{p} 21^{\mathrm{cip} 1}$ and $\mathrm{p} 27^{\mathrm{kip} 1}$. However, irradiated tumours had reduced phosphorylation of Akt, mTOR and it's target translation initiation inhibitor 4EBP1. Irradiated xenografts showed reduced microvessel density, reduced expression of CD31 but increased expression of hypoxia-induced factor 1A (HIF1a) compared to controls.

Conclusion: IR inhibits epithelial cancer tumour growth and results in sustained expression and activation of

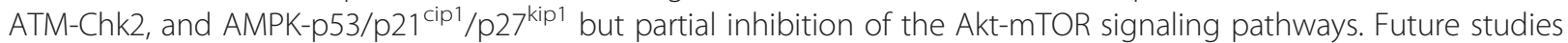
should examine causality between those events and explore whether further modulation of the AMPK and AktmTOR pathways by novel therapeutics can sensitize lung tumours to radiation.

Keywords: Lung cancer, ATM, p53, 4-EBP1, p21 ${ }^{\text {cip } 1}$

\section{Introduction}

In tumor cells ionizing radiation (IR) activates within minutes the protein kinase $\mathrm{B}$ (Akt) and mammalian Target of Rapamycin (mTOR) pathway leading to radioresistance and tumor survival [1]. Akt and mTOR are established effectors of tyrosine kinase receptors such as

\footnotetext{
* Correspondence: theos.tsakiridis@jcc.hhsc.ca

${ }^{1}$ Translational Radiation Biology Laboratory, McMaster University, Hamilton, Ontario, Canada

2Juravinski Cancer Center, McMaster University, Hamilton, Ontario, Canada Full list of author information is available at the end of the article
}

EGF receptor (EGFR), which modulates the activity of these molecules through a pathway involving phosphatidylinositol 3-kinase (PI3k) and phosphoinositidedependent kinase 1 (PDK1) [2]. Akt kinase acts as a main activator of mTOR, up regulation of which is known to occur by at least two different steps: i) phosphorylation and inhibition of Tuberous Sclerosis Complex 2 (TSC2), that inactivates GTPase activity of the GTP-binding protein Rheb leading to mTOR activation [3] and ii) stimulation of mTOR activity through phosphorylation of PRAS40, a member of mTORC1, one of

\section{Ciomed Central}


the two functional mTOR complexes, which also includes $\mathrm{mLST} 8 / \mathrm{Gbl}$ and the scaffold protein Raptor [4]. To date, extensive published work demonstrated the impact of mTOR on cell growth, cancer cell proliferation and resistance to cytotoxic agents [5] mTORC1 regulates multiple growth and gene expression pathways and specifically stimulates mRNA translation through phosphorylation and activation of the ribosomal p70S6kinase $\left(\mathrm{p} 70^{\mathrm{s} 6 \mathrm{k}}\right)$ and phosphorylation-induced inhibition of the translation initiation inhibitor eIF4E binding protein 1 (4EBP1) [5]

Recently, we showed that IR activates acutely the energy sensor and tumor suppressor AMP-activated kinase (AMPK) pathway, an evolutionally-preserved kinase that mediates a metabolic checkpoint on cell cycle when cells are under stress [6]. AMPK is an effector of Liver Kinase B 1 (LKB1), a tumour suppressor mutated in PeutzJeghers syndrome, which is associated with benign and malignant epithelial tumors [7]. AMPK is a heterotrimeric enzyme of $\alpha, \beta$ and $\gamma$ subunits that senses low energy levels through AMP binding on the $\gamma$ subunit and is regulated by phosphorylation of the $\alpha$ subunit on Thr172 [8]. AMPK inhibits anabolic processes and protein synthesis by inhibition of mTORC1 through different mechanisms including, i) Ser1387 phosphorylation and activation of TSC2, leading to enhanced Rheb GTPase activity and mTOR inhibition and ii) by Raptor phosphorylation [9]. In addition, AMPK mediates cell cycle checkpoints through induction of p53 and the cyclin-dependent kinase inhibitors (CDKI) p $21^{\text {cip } 1}$ and p $27^{\text {kip1 }}$ leading to cell cycle arrest $[6,10]$.

We have suggested that, apart from its metabolic action, AMPK is activated by IR and may be a mediator of DNA damage signals. We implicated AMPK in the mediation of IR-induced signal transduction through an Ataxia Telengiectasia mutated (ATM)-AMPK-p53p $21^{\text {cip } 1}$ pathway to facilitate $\mathrm{G} 2 / \mathrm{M}$ cell cycle arrest and mediate radiosensitization [6]. However, the effects of IR on AMPK subunit expression and chronic regulation of its activity have not been examined in human tumours. Furthermore, the levels of expression and activation of the Akt and mTOR pathways have not been analyzed extensively in irradiated tumours long after treatment. Here, we analyzed in two different human non-small cell lung cancer xenograft models the effects of a single fraction of IR on the long term expression and activation of the AMPK and the Akt-mTOR pathways, as well as their upstream regulator ATM.

\section{Methods and materials}

Animal treatments

Balb/c immune-compromised nude mice were obtained from Charles River (Mississauga, Ontario, Canada). At five weeks of age, animals were injected into the right flank with $1 \times 10^{6}$ A549 or H1299 human lung adenocarcinoma cells. Once tumours reached $100 \mathrm{~mm}^{3}$, animals were equally divided into non-irradiated (control: 0 Gy) or ionizing radiation (IR: 10 Gy) treated groups $(n=6$ per group). Tumour volume was measured every 3 days with calliper according to the formula: $\mathrm{V}=$ Lenght"Width"Height*0.5236. Eight weeks after treatment, tumours were extracted and snap-frozen in liquid nitrogen for lysis, total protein extraction and immunoblotting or were formalin fixed and paraffin embedded for immunohistochemistry (IHC) analysis. Tumour lysates were prepared from frozen tumours that were sectioned, mechanically homogenized in RIPA (Radio-Immunoprecipitation Assay) buffer and manually processed with Dounce homogenizer for total protein extraction.

\section{Animal irradiation}

After appropriate dosimetry, conformal IR treatment (10 Gy) was delivered to xenografts with a clinical radiotherapy unit while animals were anaesthetized and housed in a Plexiglas tube equipped with High-Efficiency-ParticulateAir-(HEPA) filters.

\section{Immunoblotting}

Immunoblotting was performed as described previously [6]. Antibodies for total AMPK $\alpha$, P-AMPK $\alpha$ (Thr172), P-ACC(Ser79), ATM, $\gamma$ H2AX (Ser139), P-Chk2 (Thr68), P-p53 (Ser15), p27 ${ }^{\text {kip }}, \quad$ p21 ${ }^{\text {waf/cip }}$, mTOR, P-mTOR (Ser2448), Akt, P-Akt (S473), P-Akt (Thr308), P-4EBP1 (Thr37/46), CD31 and HIF1 $\alpha$ were purchased from Cell Signalling Technology (Mississauga, Ontario, Canada). Antibodies against p53 and $\beta$-actin were supplied by Millipore (Etobicoke, Ontario, Canada).

\section{Immunohistochemistry}

Four $\mu \mathrm{m}$ thick tumour sections were mounted onto slides, deparaffinised, followed by antigen retrieval, blocking with goat serum and incubated with primary antibody against P-AMPK $\alpha$ (Thr172) (1:200), anti-CD31 (1/500) dilution overnight and processed as described earlier [11].

\section{Statistical analysis}

Quantitation and normalization of immunoblotting results was pursued for all xenograft lysates and antibodies (12 per tumour type and 6 per condition, Control vs irradiated). All density values of each immublotting band were first normalized against a value that for each blot was defined by the average density of the 6 control (untreated) lysates in each tumor type. Mean and SE values were determined after this normalization.

Paired t-test was performed to analyze the results from immunoblotting experiments using SPSS software (SPSS, 
Chicago, IL). Results are presented as Mean \pm SEM. Statistical significance was determined at $\mathrm{p}<0.05\left(^{*}\right)$.

\section{Results}

\section{Effects of IR on lung cancer xenograft growth}

Within 15 days after IR treatment, xenografts began to show differences in growth kinetics that became statistically significant by day 25 (Figure 1). At the end of the 8 week period irradiated tumours were on average $67 \pm 3.4 \%$ (A549) and $70 \pm 4.2 \%$ (H1299) smaller than their control (non-irradiated) counterparts.

\section{Effects of IR on the ATM expression and activity}

We examined the effects of IR on the total protein levels and the activity of ATM. Eight weeks after IR treatment A549 xenografts exhibited significantly increased levels of total ATM protein (Figure 2a). To evaluate the activity of ATM we assessed the phosphorylation levels of two established targets of this kinase, histone $\mathrm{H} 2 \mathrm{AX}$ and the checkpoint kinase Chk2. In both A549 and H1299 xenografts we detected increased levels of phosphorylated $\mathrm{H} 2 \mathrm{AX}(\gamma \mathrm{H} 2 \mathrm{AX})$ in the irradiated tumours compared to untreated control tumours that were significantly higher in H1299 xenografts (Figure 2A-B). Similarly, irradiated A549 and H1299 xenografts showed increased Chk2 phosphorylation (P-Chk2). That was statistically significant in $\mathrm{H} 1299$ but not in A549 xenografts when all tumours were analyzed (Figure 2).

\section{Chronic regulation of expression and activity of AMPK by IR}

In recent studies with tissue cultures of A549 cells, we observed that within 24-48 h IR stimulates expression of AMPK subunits at both the mRNA and protein level
[25]. For that we examined here whether those effects of IR could be sustained in xenografts long after IR delivery. The levels of total AMPK $\alpha$, P-AMPK and PAcetyl CoA Carboxylase (P-ACC), a substrate of AMPK indicating AMPK kinase activity, were examined in control and irradiated A549 and H1299 tumours. Basal levels of total AMPK $\alpha$ subunit increased in irradiated xenografts along with activation of the enzyme marked by phosphorylation on Thr172 residue (Figure 3A-C). $\mathrm{P}$-ACC levels were also significantly higher in tumours collected from irradiated xenografts compared to control (Figure $3 \mathrm{~A}$ and $\mathrm{B}$ ). Figure 3B shows the quantitation results of immunoblotting experiments of 6 xenografts per group. To examine whether increased levels of P-AMPK (Thr172) signals are indeed attributed to cancer cells, rather than to the surrounding tumor microenvironment, we have performed immunohistochemistry analysis of xenografts using anti-PAMPK (Thr172) antibody (Figure 3C). In those experiments we also observed significant increases in PAMPK in irradiated tumour cells compared to controls that distributed both cytoplasm and nuclei of tumor cells of A549 origin but mainly in cytoplasm of H1299 tumour cells.

\section{Regulation of steady state levels of p53 and CDKIs by IR} To examine the effects of IR treatment on cell cycle checkpoint regulators, lysates of control and IR-treated xenografts were probed with anti-p53, P-p53 (Ser15), p2 $7^{\text {kip1 }}$ and p21 ${ }^{\text {cip1 }}$ antibodies. Figure 4A-C shows that a single fraction of IR induces a sustained significant increase, of $\mathrm{p} 27^{\mathrm{kip} 1}$ and $\mathrm{p} 21^{\mathrm{cip} 1}$ levels in irradiated A549 and H1299 tumours. We analyzed total and phosphorylated (P-) p53 levels specifically in A549

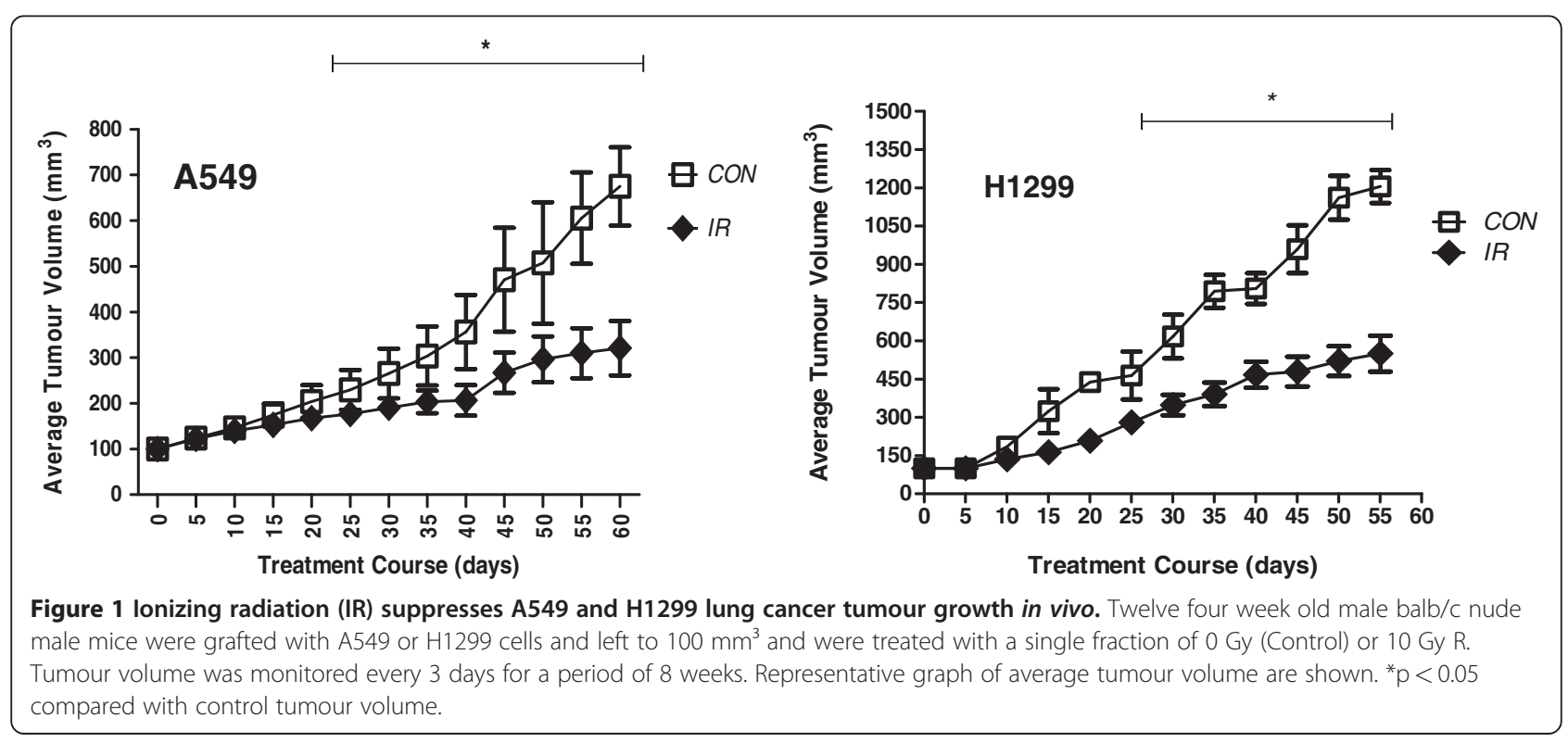




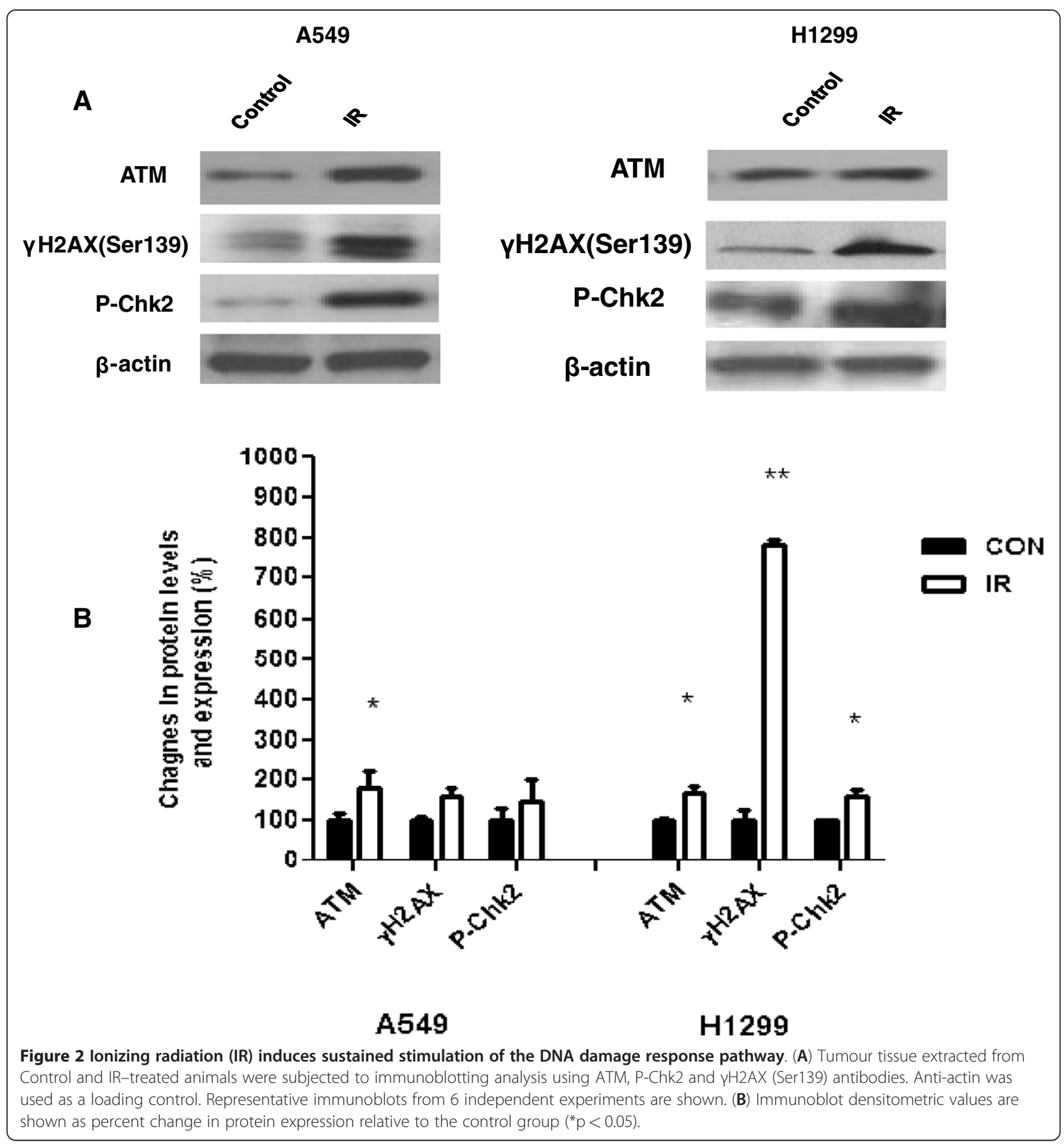

tumours only as H1299 tumours lack p53 expression. Interestingly, we detected highly significant increase in total and phosphorylated (Ser15: 5.5-fold increase) p53 levels in irradiated tumours.

IR mediates a long term suppression of the Akt-mTOR pathway

We did not detect significant differences in the total Akt levels between control and irradiated tumours
(Figure 5). However, we observed that IR caused a sustained reduction in the levels of P-AktS473 in both A549 and H1299 xenografts that reached significance in A549 but not in H1299 tumours. A trend for reduced PAktT308 levels was also detected in irradiated tumours of both types but that was not statistically significant in either of them $(30.0 \pm 6.4 \%$ and $55.0 \pm 10.9 \%$ vs $15.0 \pm 4.3 \%$ and $42.0 \pm 2.3 \%$ decrease for $\bar{T} 308$ and S473 phosphorylation in A549 and H1299, respectively) 


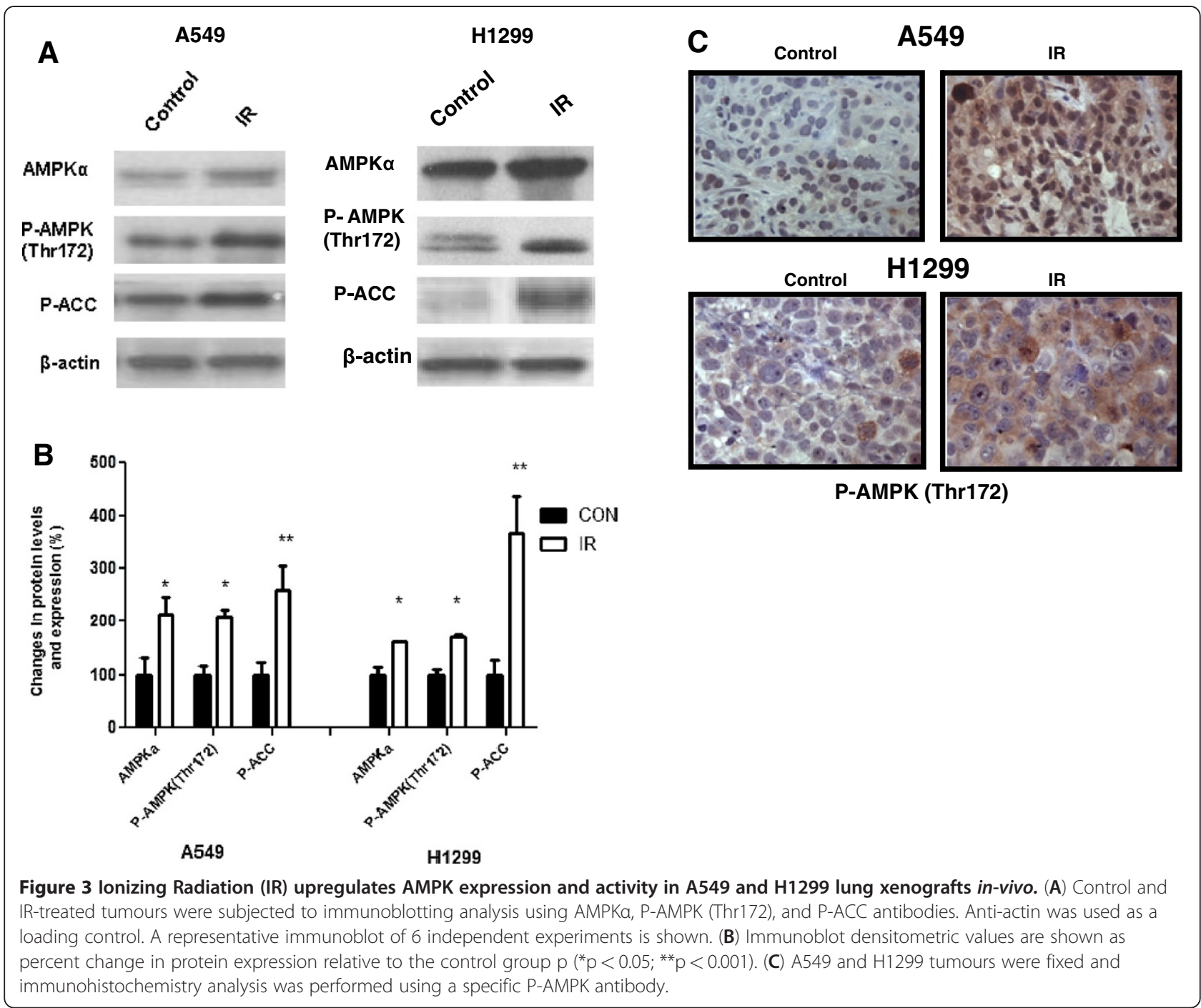

(Figure 5B, D). Consistently, both IR-treated tumour types showed reduced P-mTOR (Ser2448) levels without a significant change in total-mTOR levels. Irradiated xenografts of the two lung cancer types showed reduced levels of phosphorylation of 4EBP1 (P-4EBP1) indicating reduced mTOR activity (reduction by $81.0 \pm 4.75 \%$ and $47.0 \pm 3.20 \%$ in A549 and H1299 xenografts, respectively) (Figure 5A-B).

\section{Levels of microvasculature and hypoxia markers in irradiated xenografts}

Since hypoxia is known to modulate tumour IR responses and ATM activity, we examined the levels of the endothelial protein CD31, as a marker of microvasculature density, and those of HIF $1 \alpha$, as marker of hypoxia, in control and irradiated xenografts from both lung cancer A549 and H1299 xenografts. Figure 6A and B illustrates representative immunoblots and quantitation of results from all xenografts. Both types of irradiated xenografts showed significantly reduced levels CD31 and increased levels of HIF $1 \alpha$ in comparison to untreated tumours (Figure 6A, B). We performed immunohistochemistry experiments with the antibody against CD31 to verify whether indeed the reduced expression of CD31 levels corresponded to a reduced density of microvessels in irradiated tumours. All six tumours per group were analyzed. Figure $6 \mathrm{C}$ shows representative images from these experiments illustrating a significantly reduced density of microvessels in the irradiated A549 tumours.

\section{Discussion}

The Akt-mTOR pathway is an established mediator of radio-resistance and novel biological inhibitors of the two kinases are shown to sensitize tumour cells to IR $[12,13]$. On the other hand, AMPK is an emerging metabolic and genomic stress sensor that is also a promising target of novel cancer therapeutics such as the anti-diabetic agent metformin. Metformin inhibits 


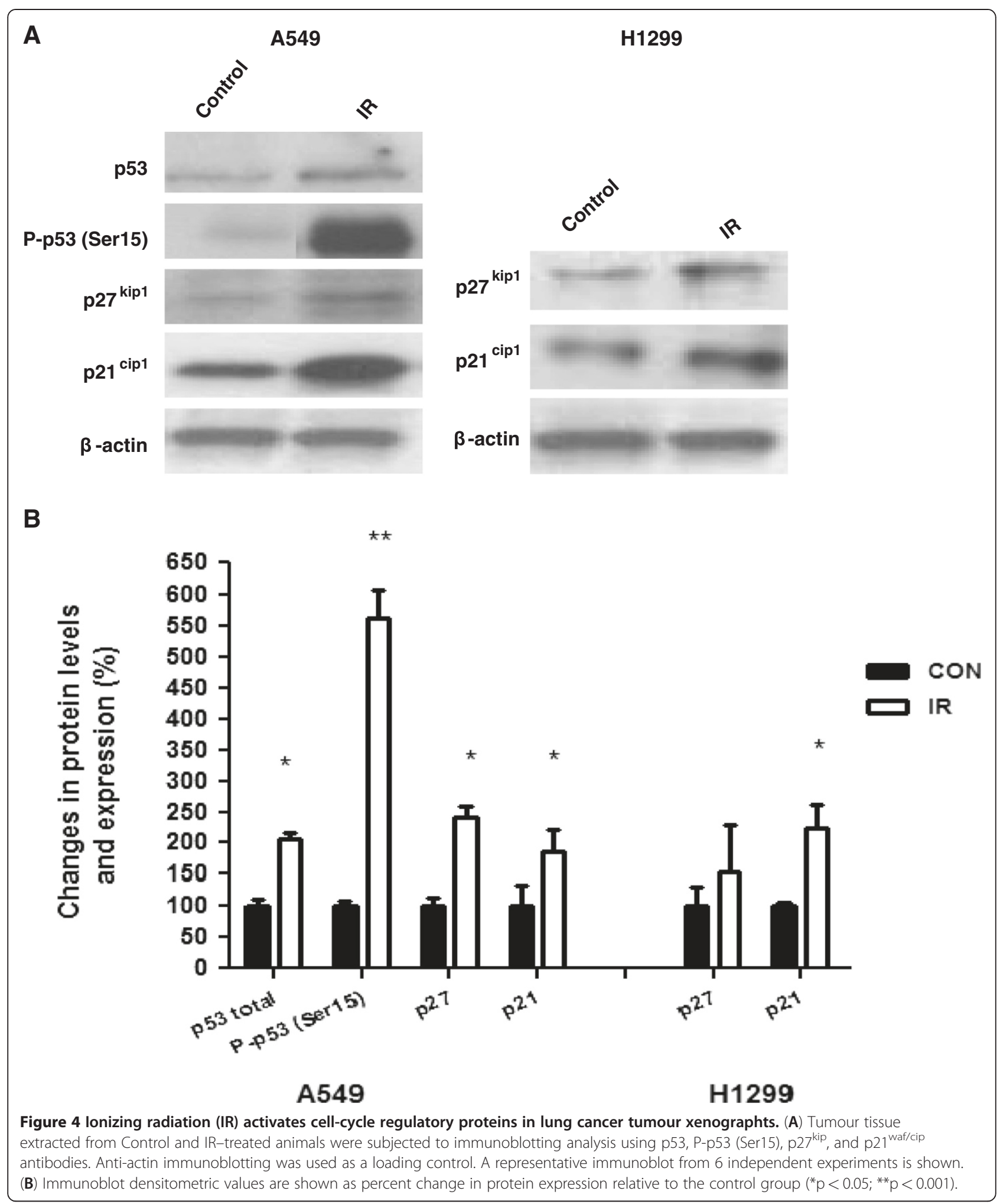

cancer cell proliferation and we have shown that it has radio-sensitizing properties in lung cancer in-vitro [6] These notions suggest a need to understand in depth the effects of IR on the expression and activity of the Akt-
mTOR and AMPK signaling pathways in tumours in order to understand better tumour radiation biology and assist in a rational development of new effective radiosensitizers. Here we analyzed the effects of a single 


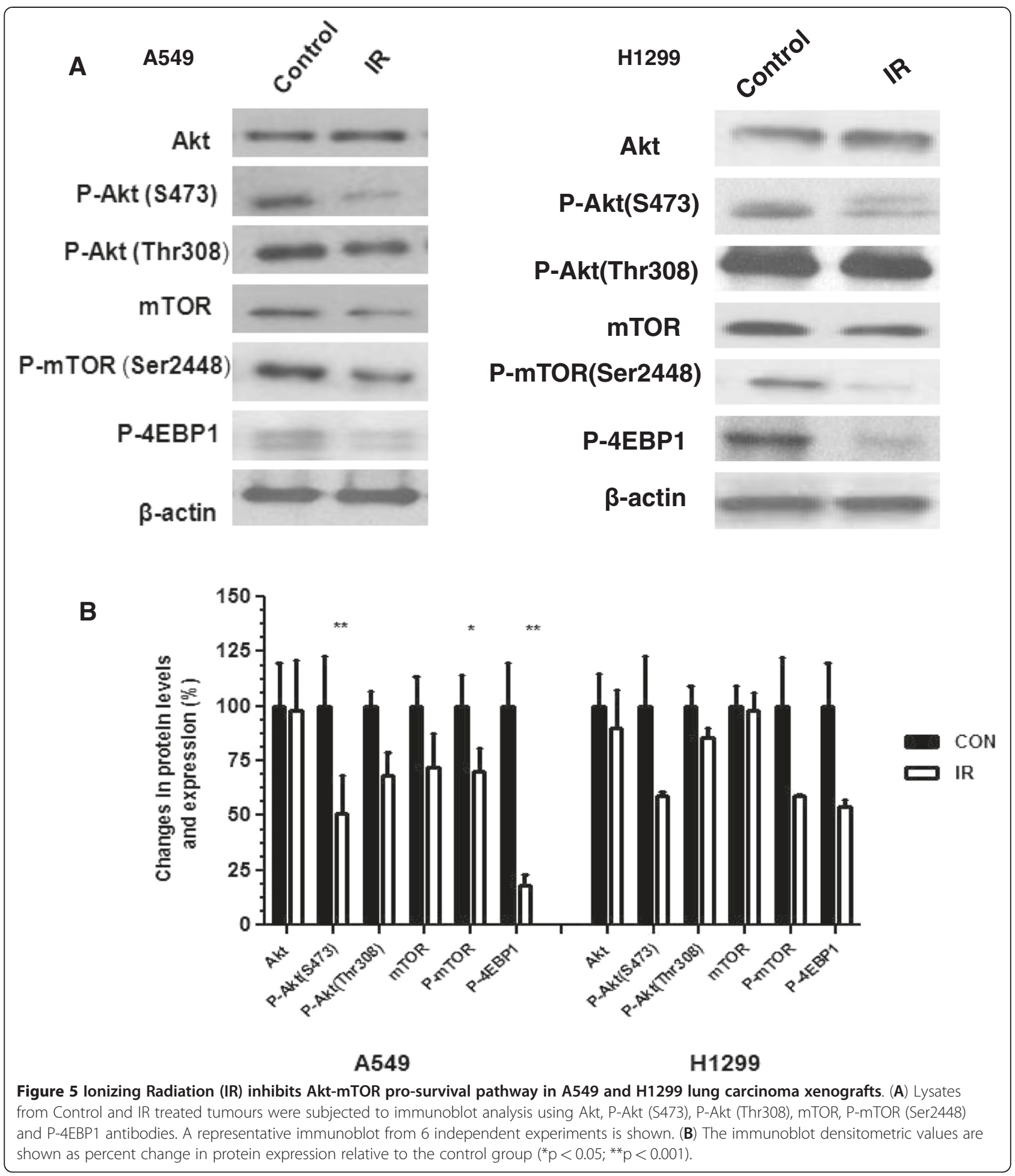

fraction of therapeutic IR (10 Gy) on the steady state levels of expression and activity of AMPK and Akt pathway members. Tumours were extracted and analyzed 8 weeks after radiation as this is a typical protocol in pre-clinical radio-sensitizer studies. Two different NSCLC tumour models with distinct molecular defects (A549:
K-Ras (G12S) oncogenic mutant and truncated LKB1-null but wild-type p53 vs H1299: p53-null, wild-type K-Ras and LKB1) were used to examine whether detected chronic response of the AMPK-p53/CDKIs and Akt-mTOR pathways to IR apply in lung cancer types with diverse oncogenic genotypes. 


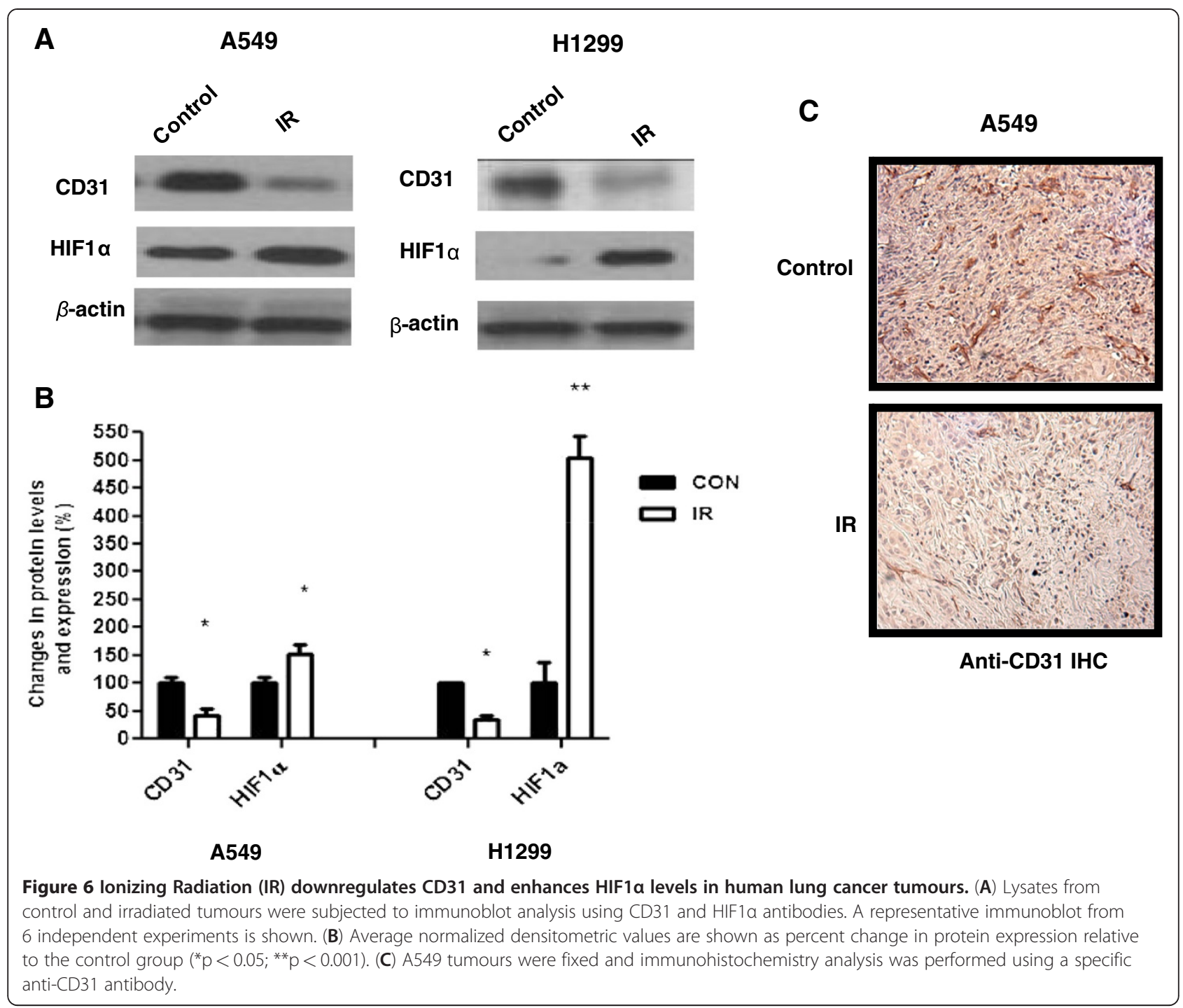

Treatment of human lung xenografts with a single fraction of IR (10 Gy) caused an expected significant inhibition of tumour growth kinetics (Figure 1). Since our earlier studies suggested that AMPK is an effector of ATM [6] and other work pointed to direct modulation of Akt activity by ATM [14] we explored the effect of IR on ATM expression and activity. Interestingly, we observed increased total ATM levels and increased phosphorylation of two ATM targets, histone H2AX and Chk2 (Figure 2). Both events are well described acute effects of IR. Enhanced levels of H2AX have also been described in human tumours $24 \mathrm{~h}$ after a clinical dose of radiotherapy of 2 Gy [15]. However, our results suggest a sustained increased activity of ATM- $\gamma \mathrm{H} 2 \mathrm{AX}$ DNA damage response pathways long after exposure to IR treatment which can be responsible for the increased activity of the AMPK pathway discussed below.
The detection of a sustained enhancement of AMPK $\alpha$ protein levels and activity in tumours long after IR is a novel finding in this study (Figure 3). Irradiated tumours had significantly higher levels of total and phosphorylated AMPK as well as P-ACC suggesting maintained enhanced expression and activity of the enzyme. Since we and others have shown that AMPK is a transducer of ATM signals $[6,16]$ sustained activation of AMPK would be an expected finding in the presence of ATM activation. However, our results also showed increased AMPK $\alpha$ protein levels, suggesting that IR drives AMPK $\alpha$ gene expression. In recent studies with lung (A549) and breast cancer cells (MCF7 and MB-231), we observed that within 24 and 48 hour IR enhances not only the activity of AMPK but also the levels of mRNA and protein of AMPK $\alpha, \beta$ and $\gamma$ subunits [17] indicating that IR regulates AMPK gene expression at both the transcriptional and the translational level. Those results suggested 
that IR stimulates significantly AMPK gene expression within $24-48 \mathrm{~h}$ that is maintained long after the genotoxic insult is delivered. The specific mechanism and transcription factors involved in these events remain to be elucidated but studies suggest involvement of the p53-dependent stress-responsive genes Sestrin 1 and 2 [18]. The regulation of AMPK gene expression and activity in response to IR is likely a universal phenomenon in epithelial tumour cells. Similar to observations in lung cancer xenografts, we have observed sustained enhancement of total and phosphorylated AMPK $\alpha$ subunit levels in xenografts of PC3 prostate cancer cells also, a cell line that lacks expression p53 (see Additional file 1: Figure S1). Therefore, overall our results suggest that IR triggers acute and chronic expression of AMPK genes as well as activation of this enzyme that is likely universal in epithelial cancer cells and is independent of p53. Currently, we analyze the exact role of sestrin genes in these processes.

Importantly, we observed that irradiated tumours maintain significantly increased levels of total and phosphorylated p53 and of CDK inhibitors p $21^{\text {cip1 }}$ and p27 $7^{\text {kip1 }}$ (Figure 4). We also detected in irradiated tumours highly increased level of p53-Ser15 phosphorylation a post-translational modification believed to contribute to a greater stability of this protein [14]. These results support the notion that IR activates the p53/ CDKI signaling pathways in tumours in a sustained fashion probably through increased expression, phosphorylation and stabilization of p53 and increased levels of CDKIs p27 $7^{\text {kip } 1}$ and $\mathrm{p} 21^{\text {cip1 }}$ (Figure 4). The p53-p21 $1^{\text {cip } 1}$ pathway is an established target for ATM [19] and AMPK $[6,8]$ both of which were suggested to phosphorylate p53. Earlier, we showed that induction of p53 and $\mathrm{p} 21^{\mathrm{cip} 1}$ in response to IR is dependent on AMPK and that AMPK activity is required for the mediation of IRinduced G2-M checkpoint and IR cytotoxicity [6]. AMPK may indeed mediate the inhibitory effects of IR on xenograft growth through regulation of p53 and CDKIs. Similar to our earlier observation on the acute response of $\mathrm{p} 21^{\text {cip } 1}$ to IR in A549 and H1299 cell cultures [6], the induction of this CDKI in irradiated xenografts does not appear to depend on p53 as it was observed in p53-null H1299 xenografts also (Figure 4 A).

IR is known to mediate a rapid activation of Akt [20] and recent studies showed that ATM can function as an activating Akt kinase that phosphorylates rapidly AktS473 [21]. Despite that, and the detection of increased ATM activity in radiated xenografts (Figure 2), we observed significantly reduced levels of Akt-S473 phosphorylation in both types of lung cancer xenografts and a trend for reduced AktT308 phosphorylation. Consistently, mTOR phosphorylation was partially reduced and so was the activity of this key enzyme indicated by lower
4EBP1 phosphorylation that was more significant in A549 tumours (Figure 5). We have obtained similar results in PC3 prostate cancer xenografts (see Additional file 1: Figure S1) indicating that these are likely universal responses of human epithelial tumours to IR that are independent of K-Ras mutation status and LKB1 or p53 function. One could contribute the suppressed mTOR activity in xenografts on the enhanced AMPK activity. However, the mechanism of reduced phosphorylation of Akt remains unclear and needs to be elucidated by future studies. Nevertheless, the concept of Akt inhibition in tumours by agents that activate the AMPK pathway has been described in earlier studies by our group and others [22,23]. It is possible that in irradiated tumours conditions develop, long after delivery of IR, that attenuate signal transduction between ATM and Akt leading to suppression of Akt and mTOR activity despite enhanced ATM activation. In irradiated tumours the combined effects of sustained increased expression

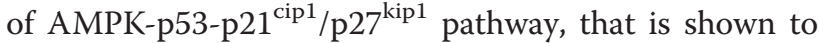
lead to inhibition of cell cycling, and inhibition of AktmTOR-4EBP1 pathway, known to lead to gene transcription and translation, may be capable of mediating an effective anti-proliferative action in those tumours, which may be adequate to mediate the cytotoxic action of IR [13]. Future studies should examine causality in the relationship between these events.

Our observation of sustained ATM activity in irradiated tumours is a significant finding of the present study. Since ATM is suggested to be a common regula-

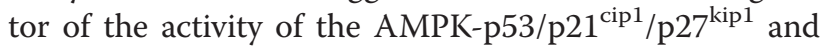
Akt-mTOR-4EBP1 pathways $[6,14]$, future work should address the mechanism of this sustained activation of ATM in irradiated tissues. It is possible that ATM activation is the result of sustained, IR-induced DNA damage or genomic instability that remains in tumours long after irradiation. Other mechanisms of ATM activation have been described, including hypoxia. Since IR is known to damage tumour vascular supply one could hypothesize that the sustained ATM activity of irradiated tumours may be the result of hypoxia developing in these tissues rather than sustained DNA damage. Conceivably, the reduced vascular supply and CD31 expression we observed in irradiated xenografts here would be responsible for local tumour hypoxia and the enhanced expression of HIF1 $\alpha$ we observed (Figure 6). Interestingly, Cam et al. [24] showed that in hypoxic conditions ATM mediates phosphorylation of HIF1 $\alpha$ leading to activation of this molecule and inhibition of mTORC1.

\section{Conclusions}

This study explored in tumours the long-term regulation by IR of two key tumour suppression or growth pathways 
that are targets of promising therapeutics. Despite established acute activation of both the AMPK and AktmTOR pathways by IR, irradiated tumours showed a sustained expression and activation of the AMPK-p53/ $\mathrm{p} 21^{\text {cip1 }} / \mathrm{p} 27^{\text {kip1 }}$ but inhibition of the activity of the AktmTOR-4EBP1 pathway. This was associated with increased expression and sustained activity of the upstream regulator of the two pathways ATM that may be associated with the development of hypoxia in irradiated tumours or with potential genomic instability. These molecular responses of irradiated tumours do not appear to be dependent on typical oncogenic molecular defects detected in lung cancer involving K-Ras, LKB1 or p53 status. The findings of this study provide a basis to understand better the chronic regulation of these key pathways by IR alone. IR causes a favorable but partial modification of the activity of the studied pathways. Additional modulation of those pathways with targeted therapies may be able to improve further radiotherapy responses in lung cancer.

\section{Additional files}

Additional file 1: Figure $\mathbf{S} 1$. The effects of ionizing radiation on $\mathrm{PC}$ prostate cancer xenografts. PC3 cells were grafted into the flanks of balb/c nude mice and were treated with or without a single fraction of $10 \mathrm{~Gy} I \mathrm{R}$. The average tumour volumes from each group were measured and expressed as the mean \pm SE of 6 animals per group. A. Extracted PC3 tumours were lysed and subjected to western blotting with antibodies against the AMPK pathway. B. Normalized densitometry values of the results from $A$. (mean \pm SE) of 6 animals per groups are shown. $\mathbf{C}$. Extracted PC3 tumours were lysed and subjected to immunoblotting with antibodies against the Akt-mTOR pathway. D. Normalized densitometry values of the results from C. (mean \pm SE) of 6 animals per groups are shown.

\section{Competing interests}

The authors declare no competing interests.

\section{Authors' contributions}

YS pursued some of the animal handling and treatments, carried out the majority of the analysis of the xenograft tissue and helped draft the manuscript. TS aided in the animal handling and treatment studies and helped draft the manuscript. SNH pursued most of the animal grafting, care and radiation treatment handling. CS helped deliver animal radiation. TF performed the radiation dosimetry study and supervised animal radiation. JCC helped optimize the immunohistochemistry protocols and reviewed slides. GS, JW and GRS provided scientific support. TT conceived the study, directed the study design, supervised all experimental work and prepared the manuscript. All authors have read and approved the final manuscript.

\section{Acknowledgements}

This work was supported by grants from the RAZCER program of the Canadian Association of Radiation Oncologists and the Prostate Cancer Canada Foundation to T.T. and the Canadian Institutes of Health Research to G.S.. We greatly appreciate the help of Dr. Eric Seidlitz on animal handling methods. We thank Dr. Robert Bristow, Princess Margaret Hospital, Toronto, ON, for scientific advice.

\section{Author details}

${ }^{1}$ Translational Radiation Biology Laboratory, McMaster University, Hamilton, Ontario, Canada. ${ }^{2}$ Juravinski Cancer Center, McMaster University, Hamilton, Ontario, Canada. ${ }^{3}$ Department of Oncology, McMaster University, 699
Concession Street, Hamilton, Ontario, Canada, L8V 5C2. ${ }^{4}$ Department of Pathology and Molecular Medicine, McMaster University, Hamilton, Ontario, Canada. ${ }^{5}$ Department of Medicine, McMaster University, Hamilton, Ontario, Canada. ${ }^{6}$ Department of Medical Physics and Applied Radiation Science, McMaster University, Hamilton, Ontario, Canada.

Received: 11 January 2012 Accepted: 08 April 2012

Published: 18 May 2012

\section{References}

1. Bussink J, van der Kogel AJ, Kaanders JH: Activation of the PI3-K/AKT pathway and implications for radioresistance mechanisms in head and neck cancer. Lancet Oncol 2008, 9:288-296.

2. Brachmann S, Fritsch C, Maira SM, Garcia-Echeverria C: PI3K and mTOR inhibitors: a new generation of targeted anticancer agents. Curr Opin Cell Biol 2009, 21:194-198.

3. Furic L, Livingstone M, Dowling RJ, Sonenberg N: Targeting mTORdependent tumours with specific inhibitors: a model for personalized medicine based on molecular diagnoses. Curr Oncol 2009, 16:59-61.

4. Wang L, Harris TE, Lawrence JC Jr: Regulation of proline-rich Akt substrate of $40 \mathrm{kDa}$ (PRAS40) function by mammalian target of rapamycin complex 1 (mTORC1)-mediated phosphorylation. J Biol Chem 2008, 283:15619-15627.

5. Laplante M, Sabatini DM: mTOR signaling at a glance. J Cell Sci 2009, 122:3589-3594.

6. Sanli T, Rashid A, Liu C, Harding S, Bristow RG, Cutz JC, Singh G, Wright J Tsakiridis T: lonizing radiation activates AMP-activated kinase (AMPK): a target for radiosensitization of human cancer cells. Int J Radiat Oncol Biol Phys 2010, 78:221-229.

7. Shackelford DB, Shaw RJ: The LKB1-AMPK pathway: metabolism and growth control in tumour suppression. Nat Rev Cancer 2009, 9:563-575.

8. Steinberg GR, Kemp BE: AMPK in Health and Disease. Physiol Rev 2009, 89:1025-1078

9. Laplante $M$, Sabatini DM: An emerging role of mTOR in lipid biosynthesis. Curr Biol 2009, 19:R1046-R1052

10. Liang J, Shao SH, Xu ZX, Hennessy B, Ding Z, Larrea M, Kondo S, Dumont DJ, Gutterman JU, Walker CL, et al: The energy sensing LKB1-AMPK pathway regulates $\mathrm{p} 27$ (kip1) phosphorylation mediating the decision to enter autophagy or apoptosis. Nat Cell Biol 2007, 9:218-224.

11. Tsakiridis T, Cutz JC, Singh G, Hirte H, Okawara G, Corbett T, Sur R, Cai W, Whelan T, Wright JR: Association of phosphorylated epidermal growth factor receptor with survival in patients with locally advanced non-small cell lung cancer treated with radiotherapy. J Thorac Oncol 2008, 3:716-722.

12. Chen H, Ma Z, Vanderwaal RP, Feng Z, Gonzalez-Suarez I, Wang S, Zhang J, Roti Roti JL, Gonzalo S: The mTOR inhibitor rapamycin suppresses DNA double-strand break repair. Radiat Res 2011, 175:214-224.

13. Konstantinidou G, Bey EA, Rabellino A, Schuster K, Maira MS, Gazdar AF Amici A, Boothman DA, Scaglioni PP: Dual phosphoinositide 3-kinase/ mammalian target of rapamycin blockade is an effective radiosensitizing strategy for the treatment of non-small cell lung cancer harboring K-RAS mutations. Cancer Res 2009, 69:7644-7652.

14. Lavin MF, Gueven N: The complexity of p53 stabilization and activation. Cell Death Differ 2006, 13:941-950.

15. Olive PL, Banath JP: Kinetics of $\mathrm{H} 2 \mathrm{AX}$ phosphorylation after exposure to cisplatin. Cytometry B Clin Cytom 2009, 76:79-90.

16. Fu X, Wan S, Lyu YL, Liu LF, Qi H: Etoposide induces ATM-dependent mitochondrial biogenesis through AMPK activation. PLOS ONE 2008, 3:e2009.

17. Sanli T, Storozhuk Y, Linher-Melville K, Bristow RG, Laderout $K$, Viollet $B$, Wright J, Singh G, Tsakiridis T: lonizing radiation regulates the expression of AMP-activated protein kinase (AMPK) in epithelial cancer cells: modulation of cellular signals regulating cell cycle and survival. Radiother Oncol 2012, 102:459-465.

18. Budanov AV, Karin M: p53 target genes sestrin1 and sestrin2 connect genotoxic stress and mTOR signaling. Cell 2008, 134:451-460.

19. Smith J, Tho LM, Xu N, Gillespie DA: The ATM-Chk2 and ATR-Chk1 pathways in DNA damage signaling and cancer. Adv Cancer Res 2010, 108:73-112. 
20. Valerie K, Yacoub A, Hagan MP, Curiel DT, Fisher PB, Grant S, Dent P. Radiation-induced cell signaling: inside-out and outside-in. Mol Cancer Ther 2007, 6:789-801.

21. Li Y, Yang DQ: The ATM inhibitor KU-55933 suppresses cell proliferation and induces apoptosis by blocking Akt in cancer cells with overactivated Akt. Mol Cancer Ther 2010, 9:113-125.

22. King TD, Song L, Jope RS: AMP-activated protein kinase (AMPK) activating agents cause dephosphorylation of Akt and glycogen synthase kinase-3. Biochem Pharmacol 2006, 71:1637-1647.

23. Sanli T, Liu C, Rashid A, Hopmans S, Tsiani E, Schultz C, Farrell T, Singh G, Wright J, Tsakiridis T: Lovastatin sensitizes lung cancer cells to ionizing radiation. Modulation of growth and tumour suppressor signalling pathways and induction of apoptosis. J Thorac Oncol. 2011, 63:439-50

24. Cam H, Easton JB, High A, Houghton PJ: mTORC1 signaling under hypoxic conditions is controlled by ATM-dependent phosphorylation of HIF1alpha. Mol Cell 2010, 40:509-520.

25. Sanli T, Storozhuk Y, Linher-Melville K, Bristow RG, Laderout K, Viollet B, Wright J, Singh G, Tsakiridis T: Ionizing radiation regulates the expression of AMP-activated protein kinase (AMPK) in epithelial cancer cells: modulation of cellular signals regulating cell cycle and survival. Radiother Oncol. 2012, 102(3):459-65.

doi:10.1186/1748-717X-7-71

Cite this article as: Storozhuk et al: Chronic modulation of AMP-Kinase, Akt and mTOR pathways by ionizing radiation in human lung cancer xenografts. Radiation Oncology 2012 7:71.

\section{Submit your next manuscript to BioMed Central and take full advantage of:}

- Convenient online submission

- Thorough peer review

- No space constraints or color figure charges

- Immediate publication on acceptance

- Inclusion in PubMed, CAS, Scopus and Google Scholar

- Research which is freely available for redistribution 